\title{
A Case of Brain Abscess Caused by the Dematiaceous Mold Neoscytalidium dimidiatum in a Korean Man
}

\author{
Su Yeon Jo $\mathbb{0}$, M.D. ${ }^{1}$, Shinwon Lee $\mathbb{C}$, M.D. ${ }^{2}$, Kye-Hyung Kim $\mathbb{1}$, M.D. ${ }^{2}$, and Jongyoun Yi $\mathbb{C}$, M.D. ${ }^{3,4}$ \\ ${ }^{1}$ Department of Laboratory Medicine, Pusan National University Yangsan Hospital, Yangsan, Korea; ${ }^{2}$ Department of Internal Medicine, Pusan National \\ University School of Medicine, Busan, Korea; ${ }^{3}$ Department of Laboratory Medicine, Pusan National University School of Medicine, Busan, Korea; \\ ${ }^{4}$ Department of Laboratory Medicine, Pusan National University Hospital, Busan, Korea
}

\section{Dear Editor,}

Neoscytalidium dimidiatum is a rapidly growing dematiaceous mold mainly found in tropical and subtropical environments. Primarily a plant pathogen, it commonly causes nail and skin infections in humans; however, very rarely it causes brain infections [1-3]. Superficial $N$. dimidiatum infections have been reported in South America, Southeast Asia, India, and Africa [4]. Additionally, there are rare reports of deep-seated infections in immunocompromised patients, with poor prognoses [1, 2, 4, 5]. Only two brain abscess cases have been reported in India and Iran [2, 3]. In Korea, this fungus is non-endemic, and N. dimidiatum human infections have not been reported. We describe a case of a brain abscess caused by $N$. dimidiatum in a Korean man who survived after prolonged antifungal therapy. The Institutional Review Board of Pusan National University Hospital, Busan, Korea, exempted the approval of this study and the need for informed consent.

This study was conducted for 15 months, from hospitalization to treatment termination and final brain imaging follow-up. A 62-year-old man with progressive dysarthria and general weakness for two weeks was admitted to a tertiary university hospital in March 2017. He had no systemic symptoms, and his underlying medical conditions included liver cirrhosis, past surgery for hepatocellular carcinoma (HCC), and diabetes. Three months earlier, he had visited Thailand. Brain magnetic resonance imaging (MRI) showed a 1.8-cm lesion; a brain abscess or HCC metastasis was suspected. Antibacterial agents were empirically administered. Stereotactic surgery was performed to aspirate the lesion, and pus was drained.

The aspirate was cultured on Sabouraud dextrose agar at $30^{\circ} \mathrm{C}$. A mold was isolated and matured quickly within three days; colonies were woolly and dark (Fig. 1A). Hyphae were septate and branched, and numerous rectangular or barrel-shaped arthroconidia were observed, with no empty cells between them (Fig. 1B). Based on these morphological features, the isolate was presumably identified as $N$. dimidiatum [1].

To genotypically confirm the species, the internal transcribed spacer (ITS) regions were sequenced [6]. The ITS1-5.8S ribosomal DNA-ITS2 sequence (GenBank accession no. MG028648) was analyzed using the International Society for Human and Animal Mycology (ISHAM) ITS database [6] and showed 99.6\% similarity to $N$. dimidiatum; Lasiodiplodia theobromae was the second most similar hit (91.0\%). The D1/D2 region of the large subunit ribosomal RNA was also sequenced [7]. Phylogenetic analysis showed the ITS was better than D1/D2 at discriminating Neoscytalidium species (Fig. 2). Matrix-assisted laser desorption/ionization time-of-flight mass spectrometry was not used for identification because the analysis could be inhibited
Received: January 28, 2020

Revision received: June 11, 2020

Accepted: September 19, 2020

Corresponding author: Jongyoun Yi, M.D., Ph.D.

Department of Laboratory Medicine, Pusan National University Hospital,

179 Gudeok-ro, Seo-gu, Busan 49241, Korea

Tel: +82-51-240-7417, Fax: +82-51-240-7413

E-mail: socioliberal@yahoo.co.kr

\section{(c) (i) (9)}

() Korean Society for Laboratory Medicine

This is an Open Access article distributed under the terms of the Creative Commons Attribution Non-Commercial License (https://creativecommons.org/licenses/by-nc/4.0) which permits unrestricted non-commercial use, distribution, and reproduction in any medium, provided the original work is properly cited. 
by the melanin pigment of the isolate [7].

After the mold was isolated, the antimicrobial regimen was altered to intravenous amphotericin B (AMB). After a 4-week AMB therapy, the brain lesion showed an approximate $30 \%$ decrease in size; however, a new 1.6-cm lesion appeared along the stereotactic aspiration tract. After two additional weeks of AMB therapy, the patient was switched to oral voriconazole and discharged. However, as the lesions remained unchanged according to the MRI conducted after 5-week voriconazole therapy, he was re-admitted and treated again with AMB for another

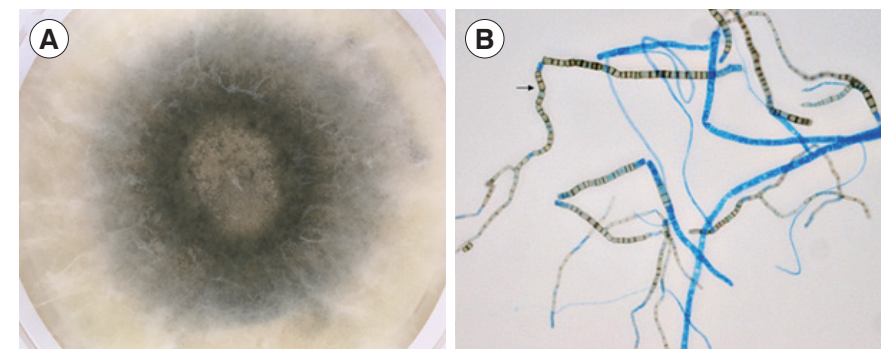

Fig. 1. Culture of the Neoscytalidium dimidiatum brain isolate on Sabouraud dextrose agar. (A) After incubation for three days, the isolate showed white woolly and dark brown to black pigmented colonies filling the agar. (B) Microscopic examination of the $N$. dimidiatum isolate $(\times 400)$. A cellophane tape mount with lactophenol cotton blue stain demonstrated septate hyphae and numerous rectangular arthroconidia (arrow). The arthroconidia and some hyphae were brown.
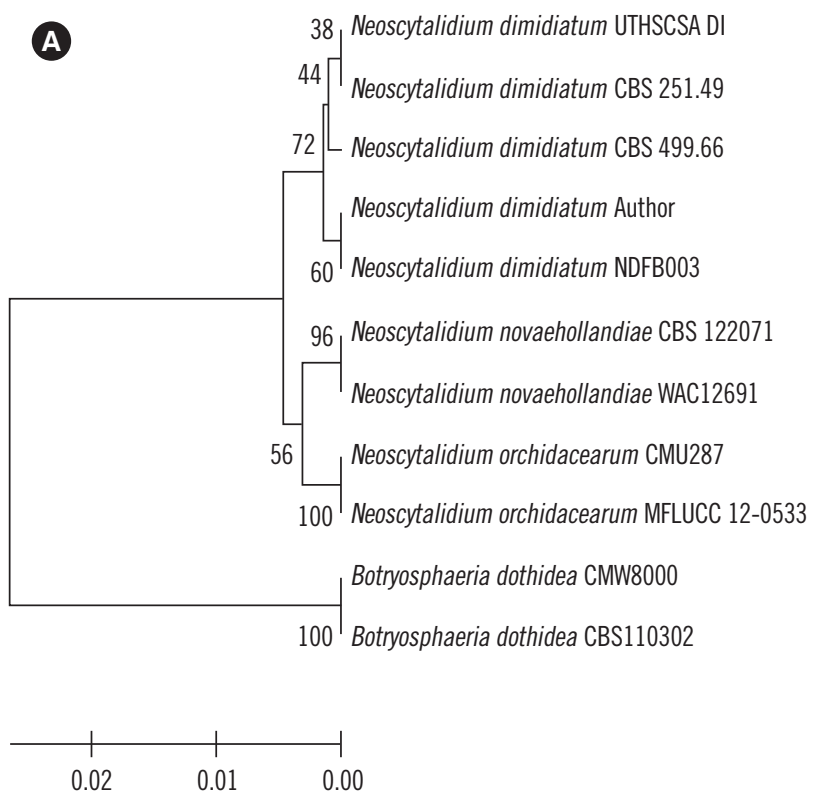

10 weeks; no further antifungals were subsequently administered. He was treated and followed-up for approximately 15 months in total. Follow-up MRIs showed steady and significant lesion improvement for one year. The dysarthria and general weakness had disappeared, and the brain abscess follow-up was concluded.

Our patient may have acquired the infection while visiting Thailand, and his immunocompromised state possibly contributed to the brain infection caused by this fungus.

Deep $N$. dimidiatum infections show poor prognoses and have no standardized treatment [5]. In vitro susceptibility analyses of $N$. dimidiatum clinical isolates from superficial infections suggest that $A M B$ and voriconazole have the highest activity and that voriconazole could constitute an alternative to AMB [8]; however, more deep-seated infections frequently show poor outcomes despite treatment with these drugs [9]. Posaconazole and caspofungin have less in vitro activity than voriconazole [8, 10]. Anidulafungin shows in vitro activity against some isolates, while micafungin demonstrates little activity [10].

We did not analyze antifungal susceptibility because it provides only minimal inhibitory concentrations, which do not correlate with clinical outcomes [8]. Nevertheless, our case indicates that voriconazole was probably ineffective because the lesions did not diminish, whereas AMB provided a gradual but obvious clinical effect. Previous brain abscess cases were fatal

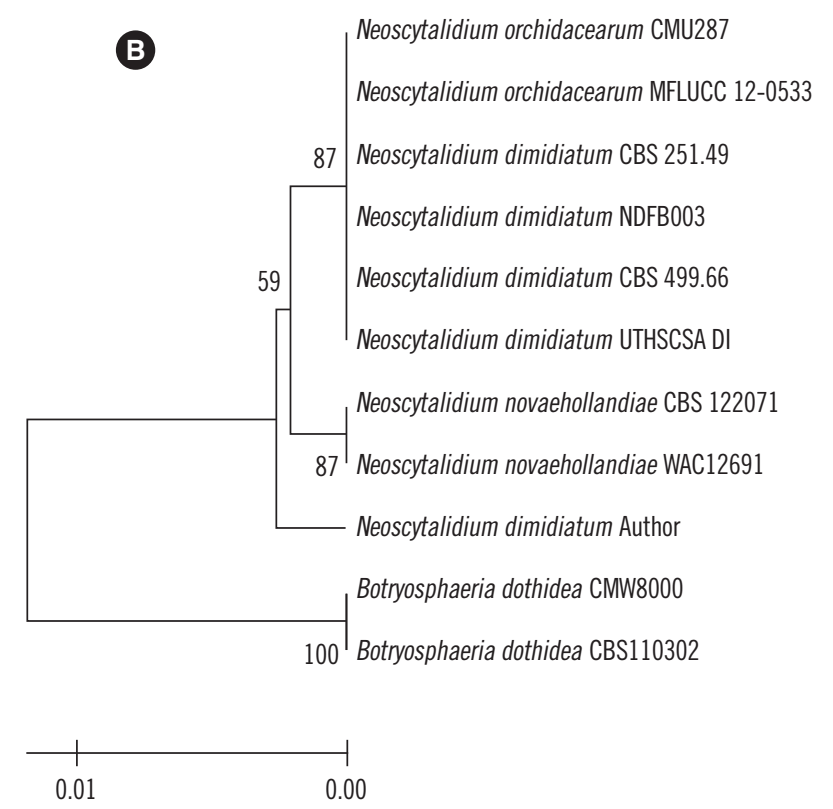

Fig. 2. Phylogenetic analysis of the isolate from this study and closely related Neoscytalidium spp. Botrysphaeria dothidea sequences were used as an outgroup. The isolate from this study is marked with 'Author' after the species name. (A) Internal transcribed spacer sequences. (B) Sequences of the D1/D2 region of large subunit ribosomal RNA. 
despite antifungal therapies [2, 3]. In contrast, our patient was eventually cured after early stereotactic drainage and 5-month antifungal therapy.

This is the first case of a brain infection by $N$. dimidiatum in Korea. Stereotactic surgery and genotypic identification using a reliable database such as ISHAM [6], rather than GenBank, could help accurately diagnose $N$. dimidiatum when an immunocompromised patient presents with brain lesions and a travel history to endemic countries. Prolonged AMB therapy can be effective in treating patients with this fungal infection, while the efficacy of voriconazole is uncertain.

\section{ACKNOWLEDGEMENTS}

None.

\section{AUTHOR CONTRIBUTIONS}

Conceptualization: JY. Data curation: JY, SYJ. Formal analysis: JY. Methodology: JY, SYJ. Investigation: SL, KHK. Writing - original draft: SYJ. Writing - review \& editing: JY, SL, KHK.

\section{CONFLICTS OF INTEREST}

No potential conflicts of interest relevant to this study are reported.

\section{RESEARCH FUNDING}

This work was supported by a 2-year research grant of Pusan National University.

\section{ORCID}

Su Yeon Jo https://orcid.org/0000-0001-7735-2897

Shinwon Lee

Kye-Hyung Kim https://orcid.org/0000-0001-7652-7093

Jongyoun Yi https://orcid.org/0000-0001-9682-9654 https://orcid.org/0000-0001-9098-3765

\section{REFERENCES}

1. Larone DH. Medically important fungi: a guide to identification. 5th ed. Washington, DC: ASM Press, 2011: 224.

2. Geramishoar M, Zomorodian K, Zaini F, Saadat F, Tarazooie B, Norouzi $M$, et al. First case of cerebral phaeohyphomycosis caused by Nattrassia mangiferae in Iran. Jpn J Infect Dis 2004;57:285-6.

3. Mani RS, Chickabasaviah YT, Nagarathna S, Chandramuki A, Shivprakash MR, Vijayan J, et al. Cerebral phaeohyphomycosis caused by Scytalidium dimidiatum: a case report from India. Med Mycol 2008;46:70511.

4. Tan DHS, Sigler L, Gibas CFC, Fong IW. Disseminated fungal infection in a renal transplant recipient involving Macrophomina phaseolina and Scytalidium dimidiatum: case report and review of taxonomic changes among medically important members of the Botryosphaeriaceae. Med Mycol 2008;46:285-92.

5. Machouart M, Menir P, Helenon R, Quist D, Desbois N. Scytalidium and scytalidiosis: what's new in 2012? J Mycol Med 2013;23:40-6.

6. Irinyi L, Serena C, Garcia-Hermoso D, Arabatzis M, Desnos-Ollivier M, Vu D, et al. International Society of Human and Animal Mycology (ISHAM)-ITS reference DNA barcoding database-the quality controlled standard tool for routine identification of human and animal pathogenic fungi. Med Mycol 2015;53:313-37.

7. CLSI. Interpretive criteria for identification of bacteria and fungi by targeted DNA Sequencing. 2nd ed. CLSI MM18. Wayne, PA: Clinical and Laboratory Standards Institute. 2018.

8. Lacroix $C$ and de Chauvin MF. In vitro activity of amphotericin B, itraconazole, voriconazole, posaconazole, caspofungin and terbinafine against Scytalidium dimidiatum and Scytalidium hyalinum clinical isolates. J Antimicrob Chemother 2008;61:835-7.

9. Elinav H, Izhar U, Benenson S, Admon D, Hidalgo-Grass C, Polacheck I, et al. Invasive Scytalidium dimidiatum infection in an immunocompetent adult. J Clin Microbiol 2009;47:1259-63.

10. Madrid H, Ruíz-Cendoya M, Cano J, Stchigel A, Orofino R, Guarro J. Genotyping and in vitro antifungal susceptibility of Neoscytalidium dimidiatum isolates from different origins. Int J Antimicrob Agents 2009;34:351-4. 